Mycologia, 97(5), 2005, pp. 1102-1110.

(C) 2005 by The Mycological Society of America, Lawrence, KS 66044-8897

\title{
Phylogeny of Rosellinia capetribulensis sp. nov. and its allies (Xylariaceae)
}

J. Bahl ${ }^{1}$

R. Jeewon

K.D. Hyde

Centre for Research in Fungal Diversity, Department of Ecology E Biodiversity, The University of Hong Kong, Pokfulam Road, Hong Kong S.A.R., P.R. China

Abstract: A new Rosellinia species, $R$. capetribulensis isolated from Calamus sp. in Australia is described. $R$. capetribulensis is characterized by perithecia immersed within a carbonaceous stroma surrounded by subiculum-like hyphae, asci with large, barrelshaped amyloid apical apparatus and large dark brown spores. Morphologically, R. capetribulensis appears to be similar to $R$. bunodes, $R$. markhamiae and $R$. megalospora. To gain further insights into the phylogeny of this new taxon we analyzed the ITS-5.8S rDNA using maximum parsimony and likelihood methods. In addition, a morphological dataset also was analyzed phylogenetically to investigate possible affinities. ITS rDNA based phylogenies reveal that $R$. capetribulensis is closely related to other Rosellinia species showing closest affinity to $R$. arcuata, $R$. necatrix and $R$. pepo. However, analysis of $R$. capetribulensis forms an unsupported branch sister to these taxa. Results from the morphological matrix indicate a close morphological affinity to members of Rosellinia subgenus Rosellinia. Despite that ITS rDNA and morphological analyses present difficulties in constructing a proper phylogenetic framework among Rosellinia and allied genera, there is sufficient evidence to support the establishment of the new taxon in the genus Rosellinia. The morphological similarities and differences between $R$. capetribulensis and allied genera such as Astrocystis and Entoleuca are also briefly discussed.

Key words: Astrocystis, Entoleuca, Nemania, phylogeny, rDNA, taxonomy, Xylariaceae, Xylariales

\section{INTRODUCTION}

The genus Rosellinia De Notaris (1844) is an important cosmopolitan genus because its species may cause many plant diseases (Whalley 1996). Members of the genus also are known to be endophytic or saprobic on a variety of plants (Petrini 1992, Smith and Hyde 2001). For over a decade, the

Accepted for publication 31 Aug 2005.

${ }^{1}$ Corresponding author. E-mail: jbahl@hkusua.hku.hk research of the fungi occurring on palms has shown this particular substrate to be a source of fungal diversity (Fröhlich and Hyde 2000, Taylor and Hyde 2003). In continuing studies, we discovered saprobic fungi on fronds of various palm species (i.e., Archontopheonix, Calamus, Livistona) in Northern Queensland and revealed a number of unique fungi. We describe a new species in the genus Rosellinia from Calamus sp.

Most work on Rosellinia has focused on species from different geographical regions. Petrini (1992, 2003) compared Rosellinia species from temperate zones and New Zealand. Rogers et al (1987) noted the rarity of Rosellinia species in tropical rain forests of North Sulawesi, Indonesia. In studies of fungi from palm hosts, Smith and Hyde (2001) indexed twelve Rosellinia species from tropical palm hosts. Rosellinia species are not frequently isolated when compared to other xylariacieous fungi recorded from palm leaf litter. Most Rosellinia collections have been isolated from deciduous woods or dicotolydenous hosts (e.g. Martin 1967; Rogers 1979; Petrini 1992, 2003).

Taxonomic concepts of Rosellinia proposed by Petrini (1992) are followed in this study. Species are delineated based on ascospore morphology. According to Petrini (2003) this is the most stable morphological character. However, character combinations of subiculum, anamorph, stromatal characters and microscopic features of the ascus and spores are used to characterize subgenera and species groups. Rosellinia subgenus Rosellinia as restricted by Petrini (1992, 2003) is characterized by stromata developing within a false subiculum, usually uniperitheciate, hard ectostroma, entostroma reduced at the base and perithecia mostly detached from stromata wall. Contained within the stromata, asci with apical apparati staining blue in Melzer's reagent and containing unicellular ascospores or with a hyaline dwarf cell, germ slit, cellular appendages and/or mucous sheath may be present. The other subgenera are Corrugata and Calomastia (Petrini 1992). Petrini and Petrini (2005) outlined this current classification, primarily based on character combinations of spore morphology, anamorph and ascus apical apparatus.

Generic delineations of related genera within the Xylariaceae are not well defined. Amphirosellinia Y.-M.Ju, JD.Rogers, H.-M.Hsieh \& Vasilyeva, Astrocystis Berk. \& Broome, Colludiscula I.Hino \& Katum., Entoleuca Syd., Guestia GJD.Smith \& K.D.Hyde, Halorosellinia (S. Schatz) Whalley, EGB.Jones, 
K.D.Hyde \& Læssøe, Helicogermslita Lodha \& D.Hawksw., Nemania SF.Gray and Stilbohypoxylon Henn. are closely related to Rosellinia (Petrini 2003, $\mathrm{Ju}$ et al 2004). A number of characters are shared amongst these genera such as apical ring and spore morphology. The perithecia of these genera are immersed within well-developed stromatic tissue. Various authors have discussed the similarities amongst these genera (Petrini and Rogers 1986; Ju and Rogers 1990; Petrini 1992, 2003; Læssøe and Spooner 1994, Rogers and Ju 1996; Smith et al 2001; $\mathrm{Ju}$ et al 2004). In this study we will restrict our investigation to the relationship of Rosellinia subgenus Rosellinia with Astrocystis, Entoleuca and Nemania. This study also provides some preliminary insights into the natural groupings of these genera based on ITS-5.8S sequence analysis.

Petrini $(1992,2003)$ has made significant contributions to the taxonomy of Rosellina. This study also will examine the relationships of Rosellinia with other morphologically similar genera using previously published datasets based on numerical taxonomy and ITS-5.8S rDNA sequence data.

\section{MATERIALS AND METHODS}

Collection.-The current study is based on collections made during the rainy season (March 2003) from Queensland, Australia. Decayed fronds from various palm species were collected from Daintree National Park, Cape Tribulation (approximately $150 \mathrm{~km}$ north of Cairns) and examined for fungi after incubation. Ascoma contents were mounted in sterile water and viewed using an Olympus light microscope. Twenty-five ascospores, 15 asci and 15 paraphyses were measured and means calculated. Rings were tested for an amyloid reaction with Melzer's reagent and presence or absence of a sheath was determined with India ink. Attempts to isolate the fungus in pure culture were unsuccessful. Spores did germinate, but failed to grow.

DNA extraction, PCR amplification and sequence assembly.DNA was extracted from herbarium specimens using the Chelex method, modified from Walsh et al (1991) and Hirata and Takamatsu (1996). Ascospores were picked up using fine forceps, suspended in $300 \mu \mathrm{L}$ of $5 \%$ Chelex solution (Bio-Rad, Richmond, California), disrupted and content vortexed thoroughly for $1 \mathrm{~min}$. Tubes were incubated at $100 \mathrm{C}$ for $10 \mathrm{~min}$ and vortexed again $1 \mathrm{~min}$ to allow maximum disruption and incubated for an additional $5 \mathrm{~min}$ at $100 \mathrm{C}$. Contents were centrifuged at a speed of $13000 \mathrm{~g}$ for $2 \mathrm{~min}$ and the supernatant was transferred to a new tube. The sample was further incubated at the same temperature, vortexed and centrifuged and supernatant used for amplification reactions.

PCR amplification reactions consisted of $5 \mu \mathrm{L}$ Promega $10 \times$ reaction buffer with $\mathrm{MgCl}_{2}, 4 \mu \mathrm{L}$ of $2.5 \mu \mathrm{M}$ dNTPs,
$0.5 \mu \mathrm{L}$ of $0.01 \% \mathrm{BSA}, 0.5 \mu \mathrm{L}$ of $1 \% \mathrm{PVP}, 1.5 \mu \mathrm{L}$ of $10 \mathrm{mM}$ of each flanking primer, $33.7 \mu \mathrm{L}$ sterile distilled $\mathrm{H}_{2} \mathrm{O}$ and $0.3 \mu \mathrm{L}$ of $5 \mathrm{u}$ of Taq polymerase. Seven to $12 \mathrm{ng}$ of DNA was used for the reaction; the volume of distilled $\mathrm{H}_{2} \mathrm{O}$ was adjusted for a final volume of $54 \mu \mathrm{L}$. ITS4 and ITS5 primers as defined by White et al (1990) were used. PCR conditions were $3 \mathrm{~min}$ hot start $(95 \mathrm{C})$ followed by 45 cycles of $1 \mathrm{~min}$ at $95 \mathrm{C}, 50 \mathrm{~s}$ at $50 \mathrm{C}$ and $1 \mathrm{~min}$ at $72 \mathrm{C}$ followed by $10 \mathrm{~min}$ extension period at $72 \mathrm{C}$. PCR product was purified with GFX $^{\mathrm{tm}}$ purification kit (27-9602-01, Amersham Biosciences) according to the instructions provided. The purified product was sequenced using an ABI 3730 autosequencer at The Genome Research Centre, The University of Hong Kong using primers ITS4 and ITS5.

Phylogenetic analysis. - Taxa from the Xylariaceae, representing the most commonly studied genera, plus additional taxa from the Amphisphaeriaceae, were included for various analyses. Multiple alignment of the data was performed using ClustalX 1.83 with a gap penalty of 14.00 and an extension penalty of 5.66 . Manual optimization was done where necessary. Ambiguous regions were excluded from analysis unless alignments could be recoded for parsimony analysis (discussed below).

All tree searches were conducted using PAUP* $4 \mathrm{~b} 10$ (Swofford 2002), unless otherwise stated. Parsimony analysis of the morphological data set published by Petrini (1992) with the addition of the new species Rosellinia capetribulensis and Astrocystis bambusae, A. cocoes and A. mirabilis. Analysis for this data set was conducted followed Petrini (1992). Except, taxa with more than 20\% data missing were excluded from this analysis.

Maximum parsimony and maximum likelihood analyses were conducted to test the appropriate placement of the new taxa in the genus Rosellinia and extract the maximum amount of phylogenetic information from the ITS data set. Weighted parsimony, treating the gaps as a fifth character, and recoding ambiguous regions was conducted using 100 heuristic search replicates using the tree bisection reconnection swapping algorithm with random addition sequence and a random starting tree. INAASE (Lutzoni et al 2000) was used in order to recode the ambiguous regions without violating positional homology. Step matrices to account for transition/transversion ( $\mathrm{Ti} / \mathrm{Tv}$ ) ratios were created based on the data set using STMatrix (Lutzoni and Zoller 2001, Dept. of Biology, Duke University, Miadlikowska et al 2002). Four regions were subsequently recoded and were incorporated into the data set. Branching support statistics were generated by bootstrapping the dataset (100 bootstrap replicates of one heuristic search).

For the maximum likelihood search Mrmodeltest version 2.0 (Nylander 2004, http://www.ebc.uu.se/systzoo/staff/ nylander.html) was used to determine the appropriate evolutionary model for likelihood search. Bayesian searches were conducted on the program MrBayes 3.0b4 (http:// morphbank.ebc.uu.se/mrbayes/, Huelsenbeck and Ronquist 2001, Ronquist and Huelsenbeck 2003). Node support was determined by conducting a Bayesian tree search and presented as posterior probabilities. The apriori assump- 
tions of the appropriate evolutionary model as determined by Mrmodeltest 2.0 (Nylander 2004) were maintained for the Bayesian as well as maximum likelihood analyses. Maximum likelihood (ML) trees were saved and compared using likelihood optimality criteria with trees generated by parsimony analyses. Trees were statistically compared using Kishino-Hasegawa test (KH test) (Kishino and Hasegawa 1989) and nonparametric Templeton Test (Templeton 1983) as implemented in PAUP.

\section{RESULTS}

Rosellinia capetribulensis J. Bahl, R. Jeewon, \& K.D. Hyde. sp. nov.

FIGS. $1-8$

Etymology. In reference to the collection locale, Cape Tribulation, Queensland, Australia.

Stroma nigra, superficialia niger, globosa (0.82 mm, $\mathrm{n}=15)$, per subiculum perdurans, atrobrunneus (FIGS. 1-2). Asci, clavate, breviter pedicellati, unitunicati, octosporim 270-290 × 45$65 \mu \mathrm{m}(280.3 \times 54.2 \mu \mathrm{m}, \mathrm{n}=15)$, apparatu apicali bene evoluto, iodo colorem caeruleum ducens (ca. $21.3 \times 14.1 \mu \mathrm{m}, \mathrm{n}=15) \quad$ (FIGS. 3-6). Ascosporae fusiformia atrobrunneus germinali longitudinali per totam 103-155 × 15-22.5 $\mu \mathrm{m}(124 \times 17 \mu \mathrm{m}, \mathrm{n}=25)$ (Figs. 7 and 8). Paraphyses numerosae, filiformes.

Ascomata on palm rachides appear as superficial black domes with a central ostiole surrounded by persistent dark brown to black superficial hyphae (subiculum-like) within a well-developed carbonaceous stroma $(0.82 \mathrm{~mm}, \mathrm{n}=15)$ (FIGS. 1 and 2). Peridium easily separated from the stroma in fresh material. Paraphyses persistent at maturity, hypha-like, tapering slightly toward the rounded apex, ca. $4.75 \mu \mathrm{m}$ at the base and $2.5 \mu \mathrm{m}$ at tip. Asci $270-290$ $\times 45-65 \mu \mathrm{m}(280.3 \times 54.2 \mu \mathrm{m}, \mathrm{n}=15), 8$-spored, clavate, short pedicellate, apically rounded, with a large non-refractive, amyloid, barrel-shaped, subapical apparatus (ca. $21.3 \times 14.1 \mu \mathrm{m}, \mathrm{n}=15$ ) (Figs. 3-6). Ascospores 103-155 × 15-22.5 $\mu \mathrm{m}(124$ $\times 17 \mu \mathrm{m}, \mathrm{n}=25$ ), overlapping bi-seriate, fusiform, tapering slightly at the apices to rounded ends, dark brown to black at maturity, unicellular, smooth, with a germ slit running the entire length of the spore, possessing a thin, inconspicuous sheath (FIGS. 7 and 8). Gene reference nuclear encoded ribosomal DNA sequence of ITS-5.8s sequence (GenBank accession no. AY862570).

Anamorph. Unknown.

Habitat. Saprobic on decaying rachis of Calamus sp.

Specimens examined. Australia. Queensland: Cape Tribulation, Daintree National Park, decaying rachis of Calamus sp., March 2003, J. Bahl CCT008 (Holotype HKU[M] no 17499).

Phylogenetic analysis. The morphological data set
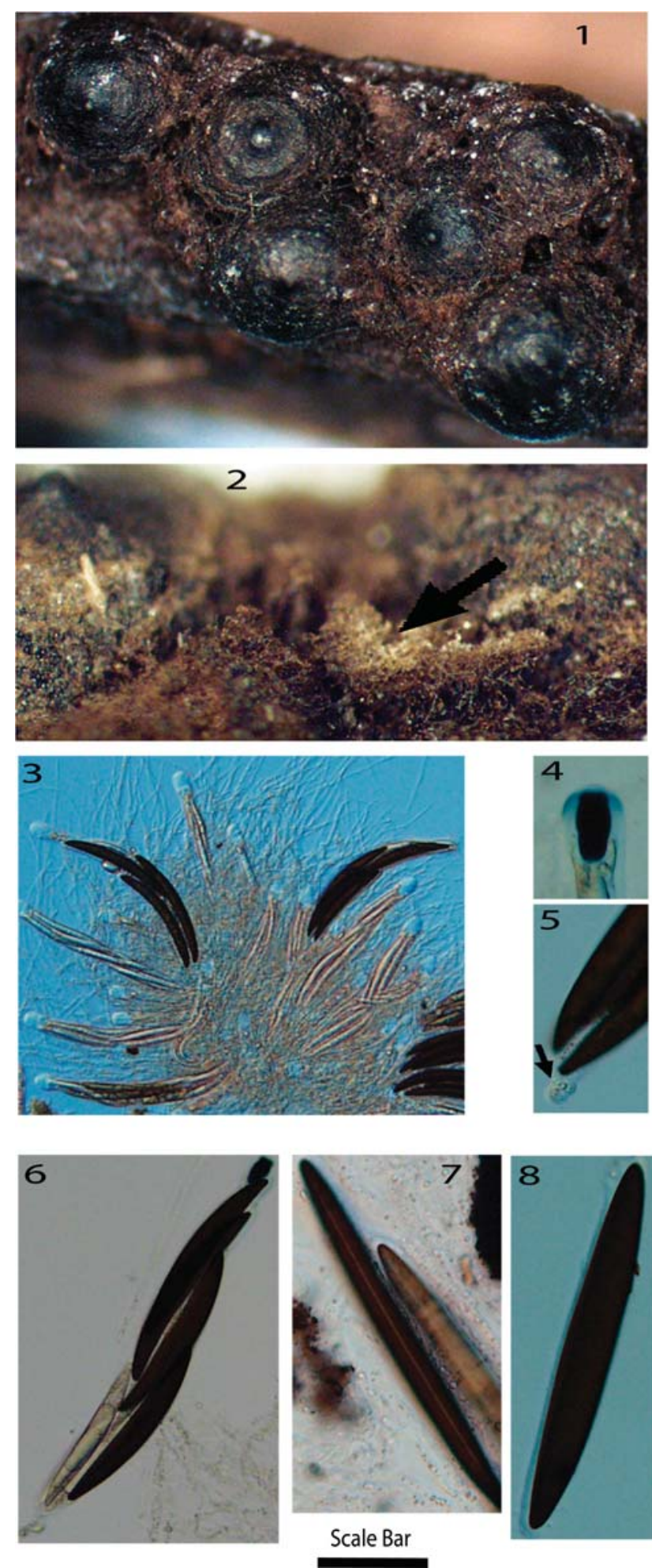

\section{Scale Bar}

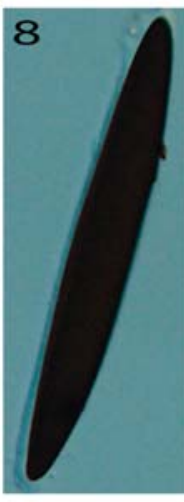

FIGS. 1-8. Rosellinia capetribulensis (from holotype). 1 Ascoma as seen on host (Calamus sp.). 2. Superficial hyphae (arrow). 3. Asci and paraphyses. 4. Asci. 5. Apical apparatus (stained in Melzer's reagent). 6. Ascus base showing the pedicel (arrow). 7. Ascospore showing thin sheath. 8 . Ascospore with germslit. Bar: $1=0.1 \mathrm{~mm}, 2=0.25 \mathrm{~mm}$, $3=90 \mu \mathrm{m}, 4=40 \mu \mathrm{m}, 5-8=25 \mu \mathrm{m}$. 


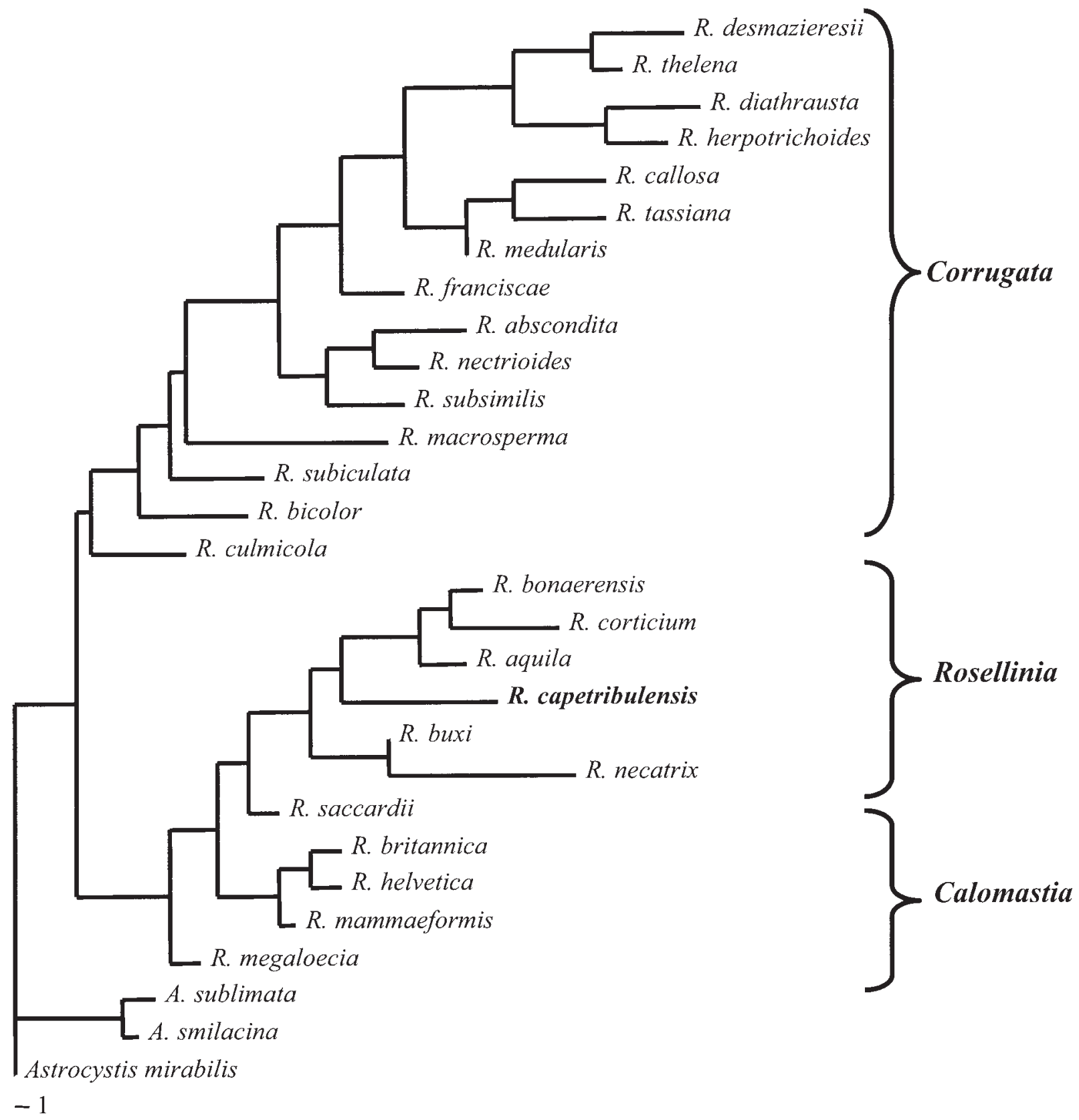

FIG. 9. One of two equally parsimonious trees generated using 31 morphological characters.

consisted of 31 characters from 35 taxa. All gaps were treated as missing and the analysis resulted in two equally parsimonious trees (FIG. 9). Both trees showed $R$. capetribulensis nested within Rosellinia subgenus Rosellinia. Apomorphic characters of each subgenus are addressed in detail in Petrini (1992). Amongst the subgenus Rosellinia, R. capetribulensis shares the following apomorphic characters: globose stromata, papillate ostiole, with cracks in the stromata, a large apical apparatus, with long and wide ascospores. Of these characters, the apical apparatus and spore length and width are unique amongst the taxa used in this analysis.

The ITS data set consisted of 722 characters from 24 taxa, of which 280 were excluded. Four regions were recoded using INNASE (Lutzoni et al 2000) and included for parsimony analysis. Phylogeny obtained from parsimony analysis (gapmode $=$ newstate; $\mathrm{Ti} / \mathrm{Tv}$ approximately $2: 3$ and 4 recoded regions) is chosen to represent the relationships of Rosellinia capetribulensis with Rosellinia subgenus Rosellinia and allies. Parsimony informative characters were approximately 
$34 \%$. The search resulted in a single tree (Fig. 10, TreeBase accession no. SN2160). Phylogeny under these criteria/parameters gave better bootstrap support for the groupings described. Bayesian values greater than $95 \%$ are shown on corresponding nodes. Rosellinia capetribulensis showed close affinities to other Rosellinia species (referred as clade A in tree). However, it formed an unsupported sister branch that was basal to this clade. Clade A includes Entoleuca mammata, which groups closely with $R$. aquila, the type of Rosellinia. Related genera Nemania and Astrocystis formed monophyletic clades respectively. However, there was little statistical support between these genera and Clade A.

Maximum likelihood searches resulted in a single tree while Bayesian searches using four chains saved 10001 trees for each Bayesian search. The first 1000 trees saved were eliminated as the burn in number for the chains was determined to be within the first 600 trees sampled. Statistical tests showed no significant differences between search treatments (results not shown). Variation of node support was the major difference between trees.

\section{DISCUSSION}

Justification for the new species. - Characters that define Rosellinia capetribulensis are: presence of a subiculum, shape and size of the stroma, the large, well-developed barrel-shaped, amyloid ascal apical apparatus, and large fusiform ascospores with a narrow sheath and a germ slit running entire length of the ascospore. These characters agree well with the taxonomic concept of Rosellinia (Ju and Rogers 1990; Petrini 1992, 2003; Læssøe and Spooner 1994; Smith and Hyde 2001). The rings are well developed, especially in taxa with large ascospores (Petrini 2003). Spore characters are most important in differentiating species (Petrini 2003), and there are few Rosellinia species that could be confused with $R$. gigantispora. Morphologically $R$. gigantispora is most similar to $R$. bunodes (Berk. and Broome) Sacc., 1882 (González and Rogers 1995) and R. megalospora (Saccas 1956). However, the ascospores of $R$. bunodes taper to fine apices (ca. 1.5-2 $\mu \mathrm{m}$ ) and have a germ slit much shorter than its spore length compared to the relatively blunt spore ends in $R$. capetribulensis and the full-length germ slit. Furthermore, in $R$. capetribulensis the ascus is clavate compared to the cylindrical the ascus of $R$. bunodes. The ascomata in $R$. capetribulensis also lack brown scale ornamentations present on ascomata of $R$. bunodes. Although the ascospores in $R$. bunodes and $R$. capetribulensis overlap in length, the spores of $R$. bunodes are 5-12 $\mu \mathrm{m}$ wide, whereas those in $R$. capetribulensis are 15-22.5 $\mu \mathrm{m}$ wide. $R$. capetribulensis and $R$. megalospora share a number of morphological characters and confusion between these taxa could arise. Primarily, the perithecia of $R$. capetribulensis are easily separated from the stroma in fresh material. The stroma of $R$. megalospora is densely gregarious and subglobose to pyriform, whereas $R$. capetribulensis is globose and sparse and sometimes in groups. The asci of both taxa are large to accommodate the large spores within and both have rounded apices and short pedicels. However, the shape of the ascus in $R$. capetribulensis is clearly clavate, whereas the asci of $R$. megalospora are cylindrical to cylindricclavate. The dimensions of the asci are 185-230 $\times$ 24-32 $\mu \mathrm{m}$ in R. megalospora compared to 270-290 $\times$ $45-65 \mu \mathrm{m}$ in $R$. gigantispora. The apical apparatus of both genera are large and amyloid and are similar in dimensions. However, the apical apparatus of $R$. megalospora yellows with age while that of $R$. capetribulensis remains hyaline at maturity. However, the apical ring of $R$. megalospora appears to show high variability in shape and dimensions at different developmental stages. Furthermore, the shape of the apical ring, although similar, appears to taper at the base whereas the apical ring of $R$. capetribulensis has similar width at the apex and base. The spores show similar morphology. The apices of ascospores in $R$. megalospora taper to finer ends compared to $R$. gigantispora, which have blunt ends. Spores of R. capetribulensis are $124 \times 17 \mu \mathrm{m}$ compared to $76.5 \times 11.6 \mu \mathrm{m}$ for taxa of $R$. megalospora. In addition, presence of a mucilagenous sheath does not seem to be a characterization of ascospores from $R$. megalospora.

The apical ring of Rosellinia capetribulensis is exceptionally large and typically barrel-shaped. The apical ring of Rosellinia subgenus Rosellinia is typically taller than broad. Shape of the ring varies slightly from cuboid to barrel shaped to slightly urn shaped. The large well-developed ring most likely facilitates the ejection of the large spores (Rogers 1979, Petrini 2003). The pedicel is rather short in $R$. capetribulensis as compared to other Rosellinia species (Smith and Hyde 2001, Petrini 2003)

Analysis of numerical data shows that $R$. capetribulensis is nested within the subgenus Rosellinia as proposed by Petrini (1992). Analyses of ITS-5.8s rDNA data concurs that this is the best placement amongst taxa sampled. Further investigations into the ITS-5.8s data set (parsimony, model based maximum likelihood) revealed little further information regarding the generic affinities of these taxa. These are explored and reviewed below. 
BAHL ET AL: ROSELLINIA CAPETRIBULENSIS SP. NOV.

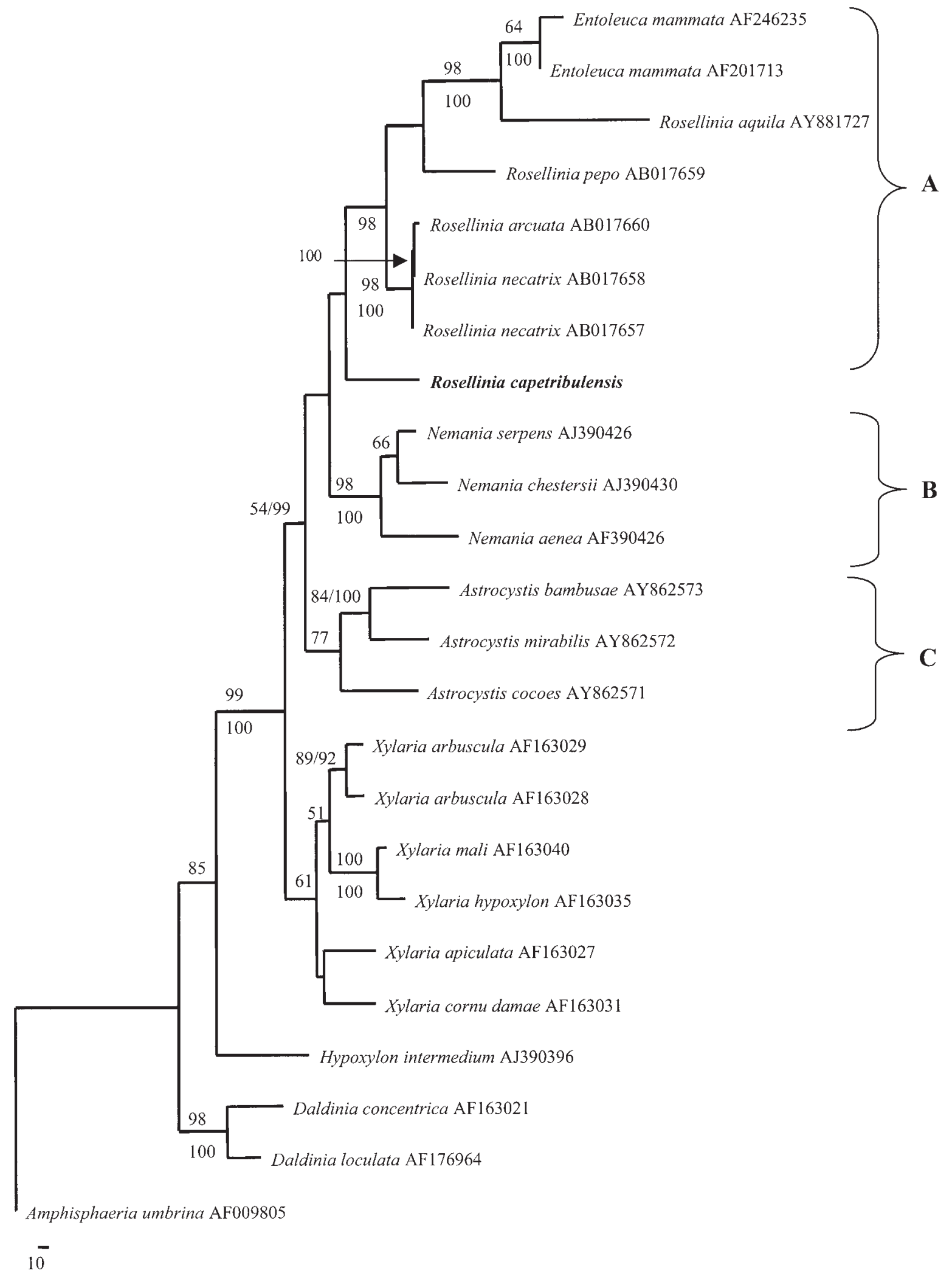

FIG. 10. Tree generated using optimality criteria based on 442 aligned characters of the ITS-5.8s regions from 24 taxa; TL $=1480.90, \mathrm{CI}=0.597, \mathrm{RI}=0.603$. Numbers represent Bootstrap (value) support (above branches) $/$ Bayesian posterior probabilities (value) (below branches or following a backslash [/]) $>50 \%$ and $>90 \%$ respectively. 
Phylogeny from the ITS sequence data contain three major clades (A, B and $\mathrm{C}$ ). Clade $\mathrm{A}$ is characterized by Entoleuca mammata and $R$. aquila, $R$. arcuata, $R$. necatrix, $R$. pepo with $R$. capetribulensis forming an unsupported sister branch. This clade is supported by high bootstrap/ Bayesian posterior probabilities. In all phylogenetic analyses, ITS sequences alone do not appear to be reliable in resolving interspecific relationships. However, analysis does indicate that Entoleuca mammata should be reassigned to Rosellinia subgenus Rosellinia. Morphologically, this is supported by the carbonaceous stroma, amyloid, apical apparatus which is taller than broad, pigmented ascospores with a straight germ slit and anamorph type. However, stromata of Rosellinia species are most frequently unitheciate and sometimes fused with neighboring stromata, but Entoleuca has multitheciate stromata. Currently, there are more than 100 Rosellinia species with a global distribution (Kirk et al 2001), and molecular data available is minimal. Analyses of the data including species available which do not fall into the subgenus Rosellinia failed to provide further information on the monophyly of the subgenus. Rosellinia desmazieri typifies the subgenus Currugata as proposed by Petrini (1992). However, inclusion into the analysis was uninformative (results not shown). Clades B and $\mathrm{C}$ are defined by the monophyletic groupings of Nemania and Astrocystis respectively. The larger clade consisting of Astrocystis, Entoleuca, Nemania and Rosellinia share a number of morphological similarities and are discussed in detail below.

Based on ITS data, $R$. capetribulensis shows affinity to members of a complex of genera that taxonomically are disputed (i.e. is Astrocystis a synonym of Rosellinia), but relationships are not statistically supported. Many of the genera grouped within this clade have at one time or another been assigned to Rosellinia. Common taxonomic characters and morphological overlap of the complex does not exclude the possibility of a common evolutionary ancestor.

Rosellinia versus Astrocystis. Astrocystis and Rosellinia are morphologically similar genera. However, differences do exist (Læssøe and Spooner 1994, Petrini 2003). Læssøe and Spooner (1994) considered differences in ascus apparatus, ascus pedicel length and the type of the anamorph to be most significant in generic delineation. The apical apparatus in Astrocystis is relatively small with parallel or slightly tapering sides. However, Rosellinia species have relatively massive and barrel-shaped apical apparati (Læssøe and Spooner 1994). The most striking difference between Astrocystis and Rosellinia is the anamorph type. In Astrocystis the anamorph has been recognized as the form genus Acanthodochium
(Samuels et al 1987, Rogers 2000), whereas Dematophora and Geniculosporium anamorphs have been assigned to Rosellinia (Rogers 1979; Petrini; 1992, 2003; Læssøe and Spooner 1994; Ju and Rogers 1990). Another difference is the presence or absence of a subiculum (Ju and Rogers 1990; Læssøe and Spooner, 1994). We follow the definition for a subiculum provided by Læssøe and Spooner (1994) where in Rosellinia species, it is actually a "false subiculum" where the ascomata are borne directly on the host tissue but surrounded by or embedded within subiculum-like hyphae rather than developing directly upon a mat of hyphae. Ju and Rogers (1990) believed that the presence or absence of a subiculum is an adaptation to the substrate and therefore not important in the generic delineation, and considered Astrocystis and Rosellinia congeneric. This relied heavily on the argument that Astrocystis bambusae and A. mirabilis belonged within Rosellinia regardless of different developmental and anamorphic characters (Ju and Rogers 1990, Rogers 2000). Phylogenetically, based on analysis of ITS, Astrocystis species forms a monophyletic clade, that is strongly supported (Clade C, 77\% bootstrap support). Due to developmental and anamorphic differences as well as the topology of the Rosellinia-complex we prefer to maintain the delineation of Astrocystis and Rosellinia.

Entoleuca and Nemania. Ju and Rogers (1990) did not accept Astrocystis as a valid genus and synonymized it with Rosellinia. Although subsequent authors did not agree (Petrini 1992, Læssøe and Spooner 1994), Rogers and Ju (1996) maintained this synonymy in their examination of Entoleuca. They believed that the type of Astrocystis, A. mirabilis had the same developmental type as Rosellinia. Petrini (1992), however, stated that the developmental type found in Astrocystis mirabilis is not seen in any species of Rosellinia. Rogers and Ju (1996) also discussed the differences between Astrocystis and Entoleuca reporting that a subiculum is lacking in the development of Entoleuca and therefore it may be similar to Astrocystis. The main difference between Astrocystis and Entoleuca is that, in Entoleuca the stroma develops below the bark of the host and is initiated by specialized coremoid pegs that break through the overlying bark, whereas in Astrocystis the ascoma develops below the anamorph and then displaces it (Rogers and Berbee 1965, Rogers and Ju 1996). Furthermore, in Entoleuca mammata (described in detail as Hypoxylon pruinatum) the anamorph develops on a sub-epidermal subiculum and not after ascomata development (Rogers and Berbee 1965). Differences between Entoleuca and Rosellinia are less clear. The teleomorphic stage of both genera is initiated below the surface of the host and the 
anamorph forms on a subiculum. However, the specialized coremoid pegs have not been described in Rosellinia and the importance of the relative position of the ascomata to the host surface (erumpent becoming superficial versus erumpent) is not clear.

We included the type of Rosellinia ( $R$. aquila) and Entoleuca mammata, which has previously been included in Hypoxylon. However, we were unable to obtain a culture of E. callimorpha, the type of the genus. In FIG. 10 Rosellinia pepo and R. aquila group with Entoleuca mammata and other Rosellinia species with $98 \%$ Bayesian posterior probability support. This indicates that Entoleuca mammata may be a species of Rosellinia. However, the multiperitheciate nature of E. mammata is considered an important character in excluding it from Rosellinia, as Rosellinia species are typically uniperitheciate (Petrini 1992, Rogers and Ju 1996). If E. mammata is synonymous with Rosellinia this suggests that the uniperitheciate definition of Rosellinia needs to be revised and that stromatal morphology of the genus might be more diverse than previously thought.

Confusion surrounds the validity of Entoleuca. Læssøe and Spooner (1994) did not accept Entoleuca as a valid genus and synonymized it with Hypoxylon. Based on comments by Miller (1961) and Martin (1967) they considered the type of the genus, Entoleuca callimorpha to be a synonym of Hypoxylon mammatum (= E. mammata). However, they did not examine the type material of E. callimorpha and suggested that H. mammatum (= E. mammata) was better placed within Rosellinia. Rogers and Ju (1996) resurrected Entoleuca based on examination of $E$. callimorpha, considered the species to be distinct from H. mammatum and transferred it to Entoleuca. Entoleuca bears a number of similarities with Nemania and Rosellinia. Developmentally, these genera are characterized by white stromatal tissue that surrounds the perithecia within the stromata. This tissue later darkens or deteriorates during maturity in many species (Rogers and Ju 1996). Furthermore these genera share a common anamorph type. The inclusion of E. callimorpha in future studies should clarify the relationship of this genus with others of the Rosellinia-complex.

Apical ring morphology appears to be of limited use in determining relationships between various other xylariaceous fungi (Læssøe and Spooner 1994, Smith and Hyde 2001). However, the apical rings of Astrocystis, Entoleuca, Nemania and Rosellinia all have rings that are typically greater in length than width. The apical ring of members of Rosellinia may be useful when associated with other characters. Astrocystis and Nemania have apical rings that closely resemble each other. However, Rosellinia tends to have apical rings that are similar in shape and dimensions to those of Xylaria when compared to the rings of Astrocystis and Nemania (Rogers and Samuels 1986, Læssøe and Spooner 1994, Smith and Hyde 2001). Furthermore, the apical ring of Entoleuca mammata is massive and more tall than broad (Rogers and Ju 1996). This character frequently occurs in species of Rosellinia.

Limited success in delineating genera within the Xylariaceae has been achieved by phylogenetic analysis using large and small subunits of the nuclear encoded ribosomal DNA (Smith et al 2003). The combination of multiple genes from different loci and morphology is needed to clarify relationships within this family. Further studies using a polyphasic approach and including data from secondary metabolites may help clarify the natural groupings of the genus Rosellinia and the putative monophyly of the genus with relation to other related taxa.

\section{ACKNOWLEDGMENTS}

J. Bahl would like to thank the University of Hong Kong for a postgraduate studentship, The Hong Kong Research Grants Council (grant no. HKU 7320/02 M) and the Committee on Research and Conference Grants (grant no. 100205773) for financial support. Michelle Nissen with the Environmental Protection Agency, Queensland, Australia is acknowledged to facilitate issue of permits required for collection, Ceri Pierce and Barbara Paulus for collection assistance and suggestions. G.J. Smith is acknowledged for providing sequence data for Astrocystis bambusae, A. cocoes, A. mirabilis and Rosellinia aquila. Cai Lei and Vijaykrishna Dhanasekaran are thanked for comments on the draft manuscript and J-M. Moncalvo for advice on phylogenetic analysis.

\section{LITERATURE CITED}

Eriksson OE, Baral H-O, Currah RS, Hansen K, Kurtzman CP, Rambold G, Læssøe T, eds. 2004. Outline of Ascomycota-2004. Myconet 10:1-99.

Fröhlich J, Hyde KD. 2000. Palm microfungi. Fung Divers Res Ser 3:1-393.

González F, Rogers JD. 1995. Rosellinia and Thamnomyces in Mexico. Mycotaxon 53:115-127.

Hirata T, Takamatsu S. 1996. Nucleotide sequence diversity of rDNA internal transcribed spacers extracted from conidia and cleistothecia of several powdery mildew fungi. Mycoscience 37:283-288.

Huelsenbeck JP, Ronquist F. 2001. MrBayes: Bayesian inference of phylogeny. Bioinformatics 17:754-755.

Ju Y-M, Rogers JD. 1990. Astrocystis reconsidered. Mycologia 82:342-349.

- 1999. The Xylariaceae of Taiwan (excluding Anthostomella). Mycotaxon 73:343-440. 
$\longrightarrow,-$ Hsieh H-M, Vasilyeva L. 2004. Amphirosellinia gen. nov. and a new species of Entoleuca. Mycologia 96:1393-1402.

Kirk PM, Cannon PF, David JC, Stalpers JA. 2001. Ainsworth $\&$ Bisby's dictionary of the fungi. 9th ed. Wallingford, UK: CAB International. $456 \mathrm{p}$.

Kishino H, Hasegawa M. 1989. Evaluation of the maximum likelihood model estimates of the evolutionary tree topologies from sequence data, and the branching order in Homonoidea. J Mol Evol 29:170-179.

Læssøe T, Spooner BM. 1994. Rosellinia \& Astrocystis (Xylariaceae): new species and generic concepts. Kew Bulletin 49:1-70.

Lutzoni F, Wagner P, Reeb V, Zoller S. 2000. Integrating ambiguously aligned regions of DNA sequences in phylogenetic analyses without violating positional homology. Syst Biol 49:628-651.

—, Zoller S. 2001. STMatrix. Program distributed by the author. Department of Biology, Duke University.

Martin P. 1967. Studies in the Xylariaceae: II. Rosellinia and the Primo-cinerea section of Hypoxylon. J S African Bot 33:315-328.

Miadlikowska J, McCune B, Lutzoni F. 2002. Pseudocyphellaria perpetua, a new lichen from Western North America. The Bryologist 105:1-10.

Miller JH. 1961. A monograph of the world species of Hypoxylon. Athens: University of Georgia Press. 158 p.

Nylander JAA. 2004. MrModeltest 2.0. Program distributed by the author. Evolutionary Biology Centre, Uppsala University.

Petrini LE. 1992. Rosellinia species of the temperate zones. Sydowia 44:169-281.

. 2003. Rosellinia and related genera in New Zealand. New Zeal J Bot 41:71-138.

—_, Petrini O, Francis SM. 1989. On Rosellinia mammaeformis and other related species. Sydowia 41:257-276.

- - 2005. Morphological studies in Rosellinia (Xylariaceae): the first step towards a polyphasic taxonomy. Mycol Res 109:569-580.

—, Rogers JD. 1986. A summary of the Hypoxylon serpens complex. Mycotaxon 26:401-436.

Rogers JD. 1979. The Xylariaceae: systematic, biological and evolutionary aspects. Mycologia 71:1-42.

- 2000. Thoughts and musings on tropical Xylariaceae. Mycol Res 104:1412-1420.

, Berbee JG. 1965. Developmental morphology of Hypoxylon pruinatum in Bark of Quaking Aspen. Phytopathology 54:154-162.
— Hypoxylon mammatum and the genus Entoleuca. Mycotaxon 59:441-448.

-, Samuels GJ. 1986. Ascomycetes of New Zealand 8. Xylaria. New Zeal J Bot 24:615-650.

— Callan BE, Samuels GJ. 1987. The Xylariaceae of the rain forests of North Sulawesi (Indonesia). Mycotaxon 29:113-172.

Ronquist F, Huelsenbeck JP. 2003. MrBayes 3: Bayesian phylogenetic inference under mixed models. Bioinformatics 19:1572-1574.

Saccas AM. 1956. Les Rosellinia des cafetiers en OubanguiChari. L'Agronomie Tropicale 11:551-614.

Samuels GJ, Rogers JD, Nagasawa E. 1987. Studies in the Amphisphaeriaceae (sensu lato) 1. Collodiscula japonica and its anamorph, Acanthodochium collodisculae. Mycotaxon 28:453-459.

Smith GJD, Hyde KD. 2001. Fungi from palms, XLIX. Astrocystis, Biscogniauxia, Cyanopulvis, Hypoxylon, Nemania, Guestia, Rosellinia and Stilbohypxylon. Fung Divers 7:89-127.

— Liew E, Hyde KD. 2003. The Xylariales: a monophyletic order containing 7 families. Fung Divers 13:175-208.

Stadler M, Ju Y-M, Rogers JD. 2004. Entonaema, Rhopalostroma, and other Xylariaceae. Mycol Res 108: 239-256.

Swofford DL. 2002. PAUP*. Phylogenetic Analysis Using Parsimony (*and other methods). version $4.0 \mathrm{~b} 10$. Sunderland, Massachusetts: Sinauer.

Taylor JE, Hyde KD. 2003. Microfungi of tropical and temperate palms. Fung Divers Res Ser 12:1-459.

Templeton AR. 1983. Phylogenetic inference from restriction endonuclease cleavage sites maps with particular reference to the evolution of humans and the apes. Evolution 37:269-285.

Walsh PS, Metzger DA, Higuchi R. 1991. Chelex 100 as a medium for simple extraction of DNA for PCR-based typing from forensic material. BioTechniques 10: 506-513.

Whalley AJS. 1996. The xylariaceous way of life. Mycol Res 100:897-922.

White TJ, Bruns TD, Lee S, Taylor J. 1990. Amplification and direct sequencing of fungal ribosomal genes for phylogenetics. In: Innis MA, Gelfand DH, Sninsky JJ, White TJ, eds. PCR protocols: A guide to methods and applications. San Diego, California: Academic Press. p 315-322. 